\title{
ARTIN ROOT NUMBERS FOR REAL CHARACTERS
}

\author{
JERRY GECHTER
}

\begin{abstract}
For $K$ and $L$ number fields, $\chi$ a real-valued character on $\operatorname{Gal}(K / L)$, the Artin root number $W(\chi)$ is \pm 1 . We analyze the question of sign for $\chi$ a degree 2 character over $\mathbf{Q}$ induced from an abelian character on a quadratic extension.
\end{abstract}

Let $K / \mathbf{Q}$ be a finite Galois extension, and $\chi$ some character on $\mathrm{Gal}(K / \mathbf{Q})$. The Artin $L$-function [2] $L(s, \chi)$ satisfies a functional equation of form $\xi(s, \chi)=W(\chi) \xi(1-s, \bar{\chi})$ where $\xi$ is $L$ multiplied by $\Gamma$ factors and (constant $)^{S}$, and $W(\chi)$ is the Artin root number associated to $\chi$. Iterating this functional equation gives $W(\chi) W(\bar{\chi})=1$. Further, if $\chi$ is real, then $W(\chi)^{2}=$ 1 or $W(\chi)= \pm 1$.

Until recently $W(\chi)$ was assumed always +1 . The first examples to the contrary were given by Serre (unpublished) and Armitage [1] using a construction of Serre. The question of sign is of considerable interest: first because an $L$-function with $W(\chi)=-1$ gives a number field whose zeta function has a zero at $\frac{1}{2}$ (see [1]), and second because the question of sign is tied up with the existence of a normal basis for the ring of integers $O_{K}$ over $\mathbf{Z}$ (see [4] and Fröhlich's Vancouver talk).

Here we consider the following set up: We start with $k$ a quadratic extension of $\mathbf{Q}$ and $\psi$ a primitive abelian character on $k$. Assume that $K$, the field defined by $\psi$ via class field theory, is normal $/ \mathbf{Q}$ and that the induced character $\chi=\psi^{*}$ on $\operatorname{Gal}(K / \mathbf{Q})$ is real valued. Then we prove:

(1) For $k$ imaginary, $W(\chi)=+1$ always.

(2) For $k$ real, tamely rarified $/ \mathbf{Q}, W(\chi)= \pm 1$, and both occur infinitely of ten with given $k$.

This result is subsumed in a recent paper of Fröhlich [5] (where $\mathbf{Q}$ is replaced by an arbitrary number field). The proof here is less elegant but more elementary.

The main idea of the proof is to look at $\psi$ restricted to $\mathbf{Z} \subset O_{k}$. This is a Dirichlet character on $\mathbf{Z}$ and in fact we have

Proposition 1. $\psi \mid \mathbf{Z}$ is a not necessarily primitive character cotrained either with the identity or with the Kronecker symbol $(d / n)$, for $d=$ discriminant of $k$.

Proof. Since $\chi=\psi^{*}$ is real, $L(s, \psi)$ has real coefficients as a Dirichlet series. That in turn says the product of the Euler factors above a single rational prime has real coefficients. Immediately we get

Received by the editors February 4, 1975.

AMS (MOS) subject classifications (1970). Primary 10H10, 12A70; Secondary 12C25.

Key words and phrases. Artin root number, Gauss sum, Artin $L$-series, normal basis.

(c) American Mathematical Society 1976 


$$
\begin{aligned}
\psi((p))= \pm 1,0 & \text { for }(d / p)=-1, \\
\psi(\underline{P})= \pm 1,0 & \text { for }(d / p)=0, \underline{P}^{2}=(p) .
\end{aligned}
$$

For $(d / p)=+1$,

$$
\begin{gathered}
(p)=\underline{P} \underline{P}^{\sigma} \quad \text { for } \sigma \in \mathrm{Gal}(k / Q) . \\
\left(1-(\underline{P}) p^{-s}\right)^{-1}\left(1-\left(\underline{P}^{\sigma}\right) p^{-s}\right)^{-1} \\
=\left(1-\left[(\underline{P})+\left(\underline{P}^{\sigma}\right)\right] p^{-s}+((p)) p^{-2 s}\right)^{-1},
\end{gathered}
$$

so $\psi(\underline{P})+\psi\left(\underline{P}^{o}\right) \in R$. Furthermore since $K$ is normal $/ \mathbf{Q}, \psi(\underline{P})$ and $\psi\left(\underline{P}^{o}\right)$ must be roots of unity of the same order. This implies

$$
\psi\left(\underline{P}^{\sigma}\right)=\overline{\psi(\underline{P})} \text {, and } \psi((p))=\psi(\underline{P}) \psi\left(\underline{P}^{\sigma}\right)=1 .
$$

We thus see that $\psi((p))=+1$ or 0 unless $(d / p)=-1$. This means $\psi \mid \mathbf{Z}$ factors through the Artin symbol

$$
\left[\frac{k / \mathbf{Q}}{p}\right]
$$

which is the statement of the proposition.

Characters $\psi$ such that $\psi \mid \mathbf{Z}$ is cotrained with the identity are precisely the classical ring class characters.

Proposition 2. Let $\psi$ be a primitive ring class character on a quadratic field. Then $W(\psi)=1$.

Proof. In [3] we explicitly evaluate the local factors in Tate's formula for $W(\psi)$. This is somewhat tedious but straightforward. Fröhlich's approach is similar. Stark (unpublished) has suggested a purely analytic proof based on the form of the functional equation for ring class characters.

THEOREM 1. If $k$ is imaginary quadratic $W(\chi)=+1$.

Proof. Since the Artin $L$-function is invariant under inducing characters, $W(\chi)=W(\psi)$. Next, since $k$ is totally imaginary, $\psi$ is unramified at $\infty$, so $\psi(-1)=+1$. By Proposition $1, \psi \mid \mathbf{Z}$ is cotrained with the identity or with $(d / n)$. For an imaginary quadratic field $(d /-1)=-1$, so $\psi$ must be cotrained with the identity. Then by Proposition $2, W(\psi)=+1$.

We now move on to the real quadratic case. Since Proposition 2 still applies, we need first to find characters $\psi$ so that $\psi \mid \mathbf{Z}$ is cotrained with $(d / n)$.

Proposition 3. If $k$ is real, tamely ramified / $\mathbf{Q}$, there exist infinitely many characters $\psi$ on $k$ satisfying

(a) $\psi$ is primitive,

(b) $\mathrm{Ker} \psi$ is invariant under conjugation,

(c) $\psi \mid \mathbf{Z}$ is cotrained with $(d / n)$.

Proof. We explicitly construct $\psi$ as a product $\psi_{p} \psi_{d}$, where $\psi_{p}$ and $\psi_{d}$ are numerical characters on $O_{k}$ (not necessarily trivial on the ray class of the fundamental unit) with the following properties: $\psi_{p}$ is defined modulo some 
rational prime $p$ and is trivial on integers; $\psi_{d} \mid \mathbf{Z}$ is $(d / n)$. In fact for $k$ tamely ramified we can take $\psi_{d}$ to be $(d / n)$ as defined on $O_{k} \bmod (\sqrt{d})$.

Let $u$ be the fundamental unit. $\psi_{d}(u)= \pm 1$. If it is +1 , then $\psi_{d}$ itself extends to a ray class character. If $\psi_{d}(u)=-1$, then we look for a rational prime $p$ and a character $\psi_{p}$ such that $\psi_{p}(u)=-1 . \psi_{p} \psi_{d}$ then will extend to a ray class character $\psi$.

The existence of the character $\psi_{p}$ is an exercise in class field theory (see [3]). That there are infinitely many $\psi$ follows since the existence proof finds infinitely many $\psi_{p}$. If $\psi$ is any character with the desired properties, $\psi_{p} \psi_{p^{\prime}}$ is another.

As yet we do not have $W(\psi)=-1$. For that we use a very special case of a theorem of Langlands (see [6], or [4] for this form). Let $\theta, \nu$ be ray class characters with conductors (finite parts) $f_{\theta}, f_{\nu}$. Let $M(\theta)$ be the number of real primes at which $\theta$ is ramified. If $\left(f_{\theta}, f_{\nu}\right)=1$ and if $M(\theta)+M(\nu)=M(\theta \nu)$ $(\bmod 4)$, then $W(\theta) W(\nu)=W(\theta \nu) \nu\left(f_{\theta}\right) \theta\left(f_{\nu}\right)$.

We now use class field theory to construct a ring class character $\psi^{\prime}$ such that

$$
\left(f_{\psi}, f_{\psi^{\prime}}\right)=1, \quad \psi\left(f_{\psi^{\prime}}\right)=-1, \quad \psi^{\prime}\left(f_{\psi}\right)=1 .
$$

Since $\psi^{\prime}$ is a ring class character it is either unramified or totally ramified at $\infty$. Further, the same is true for $\psi$ by (b) of Proposition 3. Hence the condition at the infinite primes in Langlands' theorem is satisfied. $\left(f_{\psi}, f_{\psi}\right)=1$ takes care of the other condition.

Collecting information we have $W(\psi) W\left(\psi^{\prime}\right)=W\left(\psi \psi^{\prime}\right) \psi\left(f_{\psi^{\prime}}\right) \psi^{\prime}\left(f_{\psi}\right)$ by Langlands; $W\left(\psi^{\prime}\right)=1$ by Proposition $2 ; \psi\left(f_{\psi^{\prime}}\right)=-1$ and $\psi^{\prime}\left(f_{\psi}\right)=1$ by construction. Therefore

$$
W(\psi)=-W\left(\psi \psi^{\prime}\right)
$$

$\psi \psi^{\prime}$ also satisfies (a), (b), and (c) of Proposition 3, so we have

THEOREM 2. Let $k$ be a real quadratic field, tamely ramified /Q. Then there exist infinitely many ray class characters $\psi$ on $k$ such that

(a) $\psi$ is primitive,

(b) $\mathrm{Ker} \psi$ is invariant under conjugation,

(c) $\psi \mid \mathbf{Z}$ is cotrained with $(d / n)$,

(d) $W(\psi)=-1$.

The same is true with (d) replaced by $W(\psi)=+1$.

Only condition (c) needs comment, for it shows (given (b)) that $\psi^{*}$ is a real character. To see this we reverse the reasoning in Proposition 1.

$$
\begin{aligned}
\psi^{*} \text { is real } & \Leftrightarrow \psi^{*}([(K / \mathbf{Q}) / P]) \in R \text { for all primes } P \text { of } K, \\
\Leftrightarrow & L\left(s, \psi^{*}\right) \text { has real coefficients as a Dirichlet series, } \\
\Leftrightarrow & L(s, \psi) \text { has real coefficients as a Dirichlet series, } \\
\Leftrightarrow & \psi((p))= \pm 1,0 \text { for }(d / p)=-1, \\
& \psi((p))=+1,0 \text { for }(d / p)=1,0,
\end{aligned}
$$

and this last condition follows from (c). Consequently Theorem 2 is precisely the result asserted in the case of real $k$. 


\section{REFERENCES}

1. J. V. Armitage, Zeta functions with a zero at $s=\frac{1}{2}$, Invent. Math. 20(1973), 125-138.

2. E. Artin, Zur Theorie der L-Reihen mit allgemeinen Gruppencharakteren, Hamb. Abh. 8(1930), 292-306.

3. J. Gechter, Gaussian sums for Artin L-series, Thesis, M.I.T., Cambridge, Mass., 1974.

4. A. Fröhlich, Artin root numbers and normal integral bases for quaternion fields, Invent. Math. 17(1972), 143-166. MR48 \#2115.

5. , Artin root numbers, conductors, and representations for generalized quaternion groups, Proc. London Math. Soc. 28(1974), 402-438.

6. A. Weil, Dirichlet series and automorphic forms, Lecture Notes in Math., vol. 189, SpringerVerlag, New York, 1971.

Department of Mathematics, University of Washington, Seattle, Washington 98195 